\title{
Simultaneous genome-wide association studies of anti-cyclic citrullinated peptide in rheumatoid arthritis using penalized orthogonal-components regression
}

\author{
Yanzhu Lin ${ }^{1}$, Min Zhang ${ }^{1}$, Libo Wang ${ }^{1}$, Vitara Pungpapong ${ }^{1}$, James C Fleet ${ }^{2}$ \\ and Dabao Zhang*1
}

Addresses: ${ }^{1}$ Department of Statistics, Purdue University, West Lafayette, Indiana 47907, USA and ${ }^{2}$ Department of Foods and Nutrition, Purdue University, West Lafayette, Indiana 47907, USA

E-mail: Yanzhu Lin - lin43@purdue.edu; Min Zhang - minzhang@purdue.edu; Libo Wang - wang220@purdue.edu;

Vitara Pungpapong - vpungpap@purdue.edu; James C Fleet - fleet@purdue.edu; Dabao Zhang* - zhangdb@purdue.edu

*Corresponding author

from Genetic Analysis Workshop 16

St Louis, MO, USA 17-20 September 2009

Published: 15 December 2009

BMC Proceedings 2009, 3(Suppl 7):S20 doi: 10.1186/1753-6561-3-S7-S20

This article is available from: http://www.biomedcentral.com//753-656I/3/S7/S20

(C) 2009 Lin et al; licensee BioMed Central Ltd.

This is an open access article distributed under the terms of the Creative Commons Attribution License (http://creativecommons.org/licenses/by/2.0), which permits unrestricted use, distribution, and reproduction in any medium, provided the original work is properly cited.

\begin{abstract}
Genome-wide associations between single-nucleotide polymorphisms and clinical traits were simultaneously conducted using penalized orthogonal-components regression. This method was developed to identify the genetic variants controlling phenotypes from a massive number of candidate variants. By investigating the association between all single-nucleotide polymorphisms to the phenotype of antibodies against cyclic citrullinated peptide using the rheumatoid arthritis data provided by Genetic Analysis Workshop 16, we identified genetic regions which may contribute to the pathogenesis of rheumatoid arthritis. Bioinformatic analysis of these genomic regions showed most of them harbor protein-coding gene(s).
\end{abstract}

\section{Background}

Most of the available statistical approaches for genomewide association study (GWAS) have focused on studying one single-nucleotide polymorphism (SNP) at a time [1], thereby ignoring the multigenic nature of complex diseases $[2,3]$ and the strong correlation between some SNPs due to linkage disquilibrium (i.e., some SNPs are inherited together in blocks of DNA). As pointed out by Waldron et al. [4], association studies using multiple SNPs have substantial advantages over those based on SNP associations. To capture the correlation between SNPs in regions of low recombination, haplotype-based methods that recognize the existence of linkage disequilibrium between genetic markers have been employed for multi-SNP analyses. However, such methods introduce additional problems, including the need to infer haplotypes, the impact of including (or excluding) rare haplotypes, and the need to define haplotype block boundaries. Even with haplotype-based association methods, high false-positive rates and a lack of reproducibility remain major concerns. 
Because hopes remain high for the value of GWAS, other approaches need to be pursued that account for the correlation structure among SNPs. Here we have developed a method for GWAS that incorporates regression of a phenotype simultaneously on all available SNPs, i.e., considering a multiple linear regression,

$$
\mathbf{Y}=\mu+\sum_{j=1}^{p} \beta_{j} \mathbf{X}_{j}+\varepsilon,
$$

where $\mathrm{Y}$ is the phenotypic value, $\mathrm{X}_{j}$ counts one of the two alleles at the $j^{\text {th }}$ SNP, and $\beta_{j}$ is the additive effect of that allele, $j=1, \ldots, p$. To account for the issues raised by large $p$, such as lack of independence between SNPs due to linkage disequilibrium, we will conduct the GWAS using the penalized orthogonal-components regression (POCRE) [5]. POCRE offers a fast and efficient way to identify significant SNPs simultaneously from a large number of candidates.

\section{Methods}

GWAS usually entails the collection of a massive amount of SNPs (i.e., large $p$ ) for only a small number of biological individuals (i.e., small $n$ ). Therefore, identifying the few genetic variants underlying disease risk is equivalent to the task of finding "a very few needles in a haystack", and poses a challenging statistical issue. Zhang et al. [5] recently described the POCRE approach, which sequentially constructs sparsely loaded orthogonal components with proper regularization. They demonstrate this approach works well when fitting regression models with $n<<$ data.

Let $\mathrm{Y}$ be an $n$-dimensional column phenotype vector, and $\mathrm{X}$ be an $n \times p$ genotype matrix, where $n$ and $p$ are the number of individuals and number of SNPs, respectively. Further assume both $\mathrm{Y}$ and $\mathrm{X}$ are centralized, and accordingly assume $\mu=0$ in Eq. (1). POCRE sequentially constructs orthogonal components $\tilde{\mathbf{X}}_{1} \omega_{1}, \tilde{\mathbf{X}}_{2} \omega_{2}, \ldots$, where $\tilde{\mathbf{X}}_{1}=\mathbf{X}$ and $\tilde{\mathbf{X}}_{k}, k \geq 2$ is iteratively built to be orthogonal to $\left\{\tilde{\mathbf{X}}_{1} \omega_{1}, \ldots, \tilde{\mathbf{X}}_{k-1} \omega_{k-1}\right\}$. The loading $\omega_{k}, k \geq 1$, is obtained as $\gamma /\|\gamma\|$, which minimizes

$$
-2 \gamma^{T} \tilde{\mathbf{X}}_{k}^{T} \mathbf{Y Y}{ }^{T} \tilde{\mathbf{X}}_{k} \alpha+\|\gamma\|^{2}+g_{\lambda}(\gamma), \text { subject to }\|\alpha\|=1,
$$

where $g_{\lambda}(\gamma)$ is a penalty function defined by a proper regularization on $\gamma$ with tuning parameter $\lambda$.

When $g_{\lambda}(\gamma) \equiv 0$, the optimal $\gamma$ solving Eq. (2) is proportional to the leading eigenvector of $\tilde{\mathbf{X}}_{k}^{T} \mathbf{Y Y}{ }^{T} \tilde{\mathbf{X}}_{k}$. Zhang et al. [5] employed empirical Bayes thresholding methods proposed by Johnstone and Silverman [6] to introduce proper penalty $g_{\lambda}(\gamma)$. Such penalty benefits estimating covariance between phenotype and genotypes, and provides adaptively sparse loadings of orthogonal components. The empirical Bayes implementation is also computationally efficient. The tuning parameter $\lambda$ also accounts for possible dependence structure among different SNPs, and 10-fold crossvalidation was employed to elicit its optimal values ranging from 0.8 to 1 , i.e., considering candidate values $\lambda \in\{0.8,0.82,0.84,0.86,0.88,0.9,0.92,0.94,0.96$, $0.98,1\}$.

The sequential construction of the orthogonal components stops when the optimal $\gamma$ solving Eq. (2) is zero, which implies $\tilde{\mathbf{X}}$ is hardly correlated to Y Then, regressing $\mathrm{Y}$ on the orthogonal components, i.e., $\tilde{\mathbf{X}}_{1} \omega_{1}, \tilde{\mathbf{X}}_{2} \omega_{2}, \cdots$, provides an estimate of $\beta_{1}, \ldots, \beta_{p}$ in Eq. (1). Because nonzero loadings in $\omega_{j}, j=1,2, \ldots$, are sparse, most of estimated $\beta_{1}, \ldots, \beta_{p}$ are therefore zero, reflecting the fact that most SNPs are insignificantly associated or are even completely uncorrelated to the phenotype of interest.

\section{Results}

Using our novel method (POCRE), the rheumatoid arthritis data from Genetic Analysis Workshop 16 were investigated for associations between SNPs and a serum biomarker for rheumatoid arthritis, i.e., antibodies anticyclic citrullinated peptide (anti-CCP). In this dataset, only 867 samples were positive for anti-CCP. The data set was preprocessed with the computer program PLINK [7] to control data quality, and with the computer program EIGENSTRAT [8] to control potential population structures. After preprocessing there were 490,613 SNPs remaining for the GWAS. POCRE was applied individually to each chromosome for the simultaneous association of the SNPs in that chromosome with the anti-CCP phenotype. The effects of the 10 principal components constructed by EIGENSTRAT were considered as covariates for the POCRE. Nonzero effects of SNPs were reported on seven chromosomes where the positive $\hat{\beta}$ indicates that the minor allele will increase the level of anti-CCP (Table 1).

Of the 12 SNPs identified to be associated with anti-CCP level, 5 SNPs are from chromosome 6, 2 SNPs from chromosome 12, and $1 \mathrm{SNP}$ each is from chromosomes $7,11,13,17$, and 21 , respectively. The location of each significant SNP was mapped to the human genome using the Ensembl database http://www.ensembl.org. Based on this analysis, eight of the SNPs were found to reside in seven genes (Table 1). For the other four SNPs, the nearest neighboring genes are listed in the table. None of these genes we identified have previously been linked to rheumatoid arthritis, but several of them encode proteins whose biological roles may be involved in the 
Table I: SNPs identified with nonzero coefficients in Eq. (I)

\begin{tabular}{|c|c|c|c|c|c|}
\hline SNP & $\hat{\beta}_{j}$ & Chromosome & SNP Location (bp) & Gene & Gene Location (bp) \\
\hline rs233492 & $|49.75| 4$ & $6 p 23$ & $14,910,666$ & $J A R I D 2^{\mathrm{a}}$ & $|4,754,745-| 4,754,848$ \\
\hline rs 17068819 & 109.0095 & $6 \mathrm{q} 21$ & $108,246,263$ & SCML4 & $108,|30,060-108,252,2| 4$ \\
\hline rs 922898 & 56.7294 & $6 q 25.1$ & $149,155,750$ & UST & $|49||| 0,,165-|49,439,8| 8$ \\
\hline rs692940I & 83.7685 & $6 q 25.1$ & $149,158,549$ & UST & $|49| ,1|0,165-| 49,439,8 \mid 8$ \\
\hline rs 17087579 & 187.2452 & $6 \mathrm{q} 25.1$ & $149,886,810$ & PPIL4 & $|49,867,324-| 49,908,864$ \\
\hline rsII760836 & 358.7046 & $7 p 21.2$ & $14,903,193$ & $D G K B$ & $|4,15|, \mid 99-14,909,359$ \\
\hline rs II 029744 & 300.2939 & ||$p \mid 4.2$ & $26,909,033$ & ACO 16450.10 & $26,972,204-26,975,206$ \\
\hline rs 10861038 & 63.7626 & $12 q 23.3$ & $102,455,524$ & $S T A B 2^{\mathrm{a}}$ & $|02,505| ,8|-| 02,684,635$ \\
\hline rs $10507 \mid 67$ & || $7.538 \mid$ & $12 \mathrm{q} 23.3$ & $102,490,212$ & $S T A B 2^{a}$ & $|02,505| ,8|-| 02,684,635$ \\
\hline rs 17055893 & 265.3688 & $13 q \mid 3.3$ & $36,961,628$ & POSTN ${ }^{\mathrm{a}}$ & $37,034,779-37,070,874$ \\
\hline rs 2322047 & 324.3976 & $17 p 12$ & $|1,556,5| 2$ & DNAH9 & ||$, 442,473-||, 8 \mid 3,856$ \\
\hline rs9305833 & 102.7568 & $2 \mid q 21.1$ & $18,238,917$ & CHODL & $|8| 95,,45|-| 8,56|, 56|$ \\
\hline
\end{tabular}

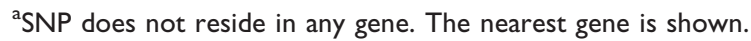

pathogenesis of this disease. For example, two SNPs (rs922898, rs6929401) that reside in the uronyl-2sulfotransferase (UST) gene were identified by our method. UST is involved in the chondroitin 3 sulfate and glycan structure biosynthesis pathways and could contribute to optimal cartilage development or repair. PPIL4 is a member of cyclophilin family, a group of proteins crucial for protein folding and immunosuppression by cyclosporin A (CsA) [9]. Polymorphisms in this gene may influence development of inflammation during rheumatoid arthritis or the response of individuals with rheumatoid arthritis to treatment. DGKB encodes diacylglycerol kinase and participates in intracellular signalling processes via several pathways including the protein kinase $\mathrm{C}$ pathway [10]. Alteration in intracellular signalling could negatively influence inflammatory processes. Finally, CHODL encodes a type I transmembrane protein including a single carbohydrate recognition domain for the C-type lectins that can worsen inflammation [11].

\section{Discussion}

In addition to its diagnostic value for the general risk of rheumatoid arthritis, a high anti-CCP level is associated with a high risk of developing joint damage during the disease. As a result, an anti-CCP-test has been employed to monitor the progression of the disease. With our novel approach for GWAS, we identified several candidate SNPs associated with the level of anti-CCP in rheumatoid arthritis patients. Among the candidate genes identified, only PPIL4 is functionally related to the immune system. Further investigation will be necessary to define the potential roles that the other gene products play in rheumatoid arthritis. Due to the small sample size available for this study (867) and the large total number of SNPs measured $(490,613)$, we expect some of the associations we identified to constitute false positives. Determining false detection rates will require development of additional procedures.

\section{Conclusion}

Our analyses using the newly developed method POCRE indicate that the genomic region 6q25.1 may harbor genes associated with anti-CCP level in rheumatoid arthritis patients. Further investigation is necessary to confirm this observation.

\section{List of abbreviations used}

anti-CCP: Anti-cyclic citrullinated peptide; GWAS: Genome-wide association study; POCRE: Penalized orthogonal-components regression; SNP: Single-nucleotide polymorphism.

\section{Authors' contributions}

YL and MZ designed the study, and YL performed the statistical analysis. MZ and DZ both conceived the study, and drafted the manuscript. LW and VP participated in the design of the study and preprocessing of the data. JCF participated in interpreting the statistical analysis results, reviewing and editing the manuscript. All authors read and approved the final manuscript.

\section{Acknowledgements}

The Genetic Analysis Workshops are supported by NIH grant ROI GM03I575 from the National Institute of General Medical Sciences. Support from the CCE project at the Oncological Sciences Center of Purdue University is gratefully acknowledged. This research was also partially supported by NSF grant IIS 0844945 to DZ, Purdue Research Foundation grant to $M Z$, and Purdue Discovery Park seed grant to $M Z$ and DZ.

This article has been published as part of BMC Proceedings Volume 3 Supplement 7, 2009: Genetic Analysis Workshop 16. The full contents of the supplement are available online at http://www.biomedcentral.com/ $|753-656| / 3$ ? issue $=$ S7.

\section{References}

I. Balding DJ: A tutorial on statistical methods for population association studies. Nat Rev Genet 2006, 7:78I-79I.

2. Pritchard $\mathrm{JK}$ and Rosenberg NA: Use of unlinked genetic markers to detect population stratification in association studies. Am J Hum Genet 1999, 65:220-228. 
3. Sham P, Bader JS, Craig I, O'Dorrovan M and Owen M: DNA pooling: a tool for large-scale association studies. Nat Rev Genet 2002, 3:862-87I.

4. Waldron ERB, Whittaker JC and Balding DJ: Fine mapping of disease genes via haplotype clustering. Genet Epidemiol 2006, 30:170-179.

5. Zhang D, Lin $Y$ and Zhang M: Penalized orthogonal-components regression for large p small $\boldsymbol{n}$ Data. Electron J Stat 2009, 3:78I-796.

6. Johnstone IM and Silverman BW: Needles and straw in haystacks: empirical Bayes estimates of possibly sparse sequences. Ann Stat 2004, 32: I594-1649.

7. Purcell S, Neale B, Todd-Brown K, Thomas L, Ferreira MAR, Bender D, Maller J, Sklar P, de Baker PIW, Daly MJ and Sham PC: PLINK: A tool set for whole-genome association and population-based linkage analysis. Am J Hum Genet 2007, 8I:559-575.

8. Price AL, Patterson NJ, Plenge RM, Weinblatt ME, Shadick NA and Reich D: Principal components analysis corrects for stratification in genome-wide association studies. Nat Genet 2006, 38:904-909.

9. Wang P, Cardenas M, Cox G, Perfect J and Heitman J: Two cyclophilin A homologs with shared and distinct functions important for growth and Cryptococcus neoformans. EMBO Rep 200I, 2:5II-5I8.

10. Goto $\mathrm{K}$ and Kondo $\mathrm{H}$ : Molecular cloning and expression of a 90-kDa diacylglycerol kinase that predominantly localizes in neurons. Proc Natl Acad Sci USA 1993, 90:7598-7602.

1I. Weng L, Smits P, Wauters J and Merregaert J: Molecular cloning and characterization of human chondrolection, a novel type I transmembrane protein homologous to C-type lections. Genomics 2002, 80:62-70.

\section{Publish with Biomed Central and every scientist can read your work free of charge}

"BioMed Central will be the most significant development for disseminating the results of biomedical research in our lifetime. "

Sir Paul Nurse, Cancer Research UK

Your research papers will be:

- available free of charge to the entire biomedical community

- peer reviewed and published immediately upon acceptance

- cited in PubMed and archived on PubMed Central

- yours - you keep the copyright
BioMedcentral 